\title{
Furfuryl alcohol/tung oil matrix-based composites reinforced with bacterial cellulose fibres
}

\author{
Henrique Augusto Silva Valentino • Paulo de Tarso Laia dos Reis e Silva Pupio • \\ Alessandro Gandini · Talita M. Lacerda (i)
}

Received: 11 February 2021 / Accepted: 10 June 2021 / Published online: 17 June 2021

(C) The Author(s), under exclusive licence to Springer Nature B.V. 2021

\begin{abstract}
Polymeric materials have drastically changed the society in the last century. However, their nonrenewable origin, together with their indiscriminate use and disposal, resulted in a huge accumulation of waste in the environment and raised a wide discussion about the emission of greenhouse gases, which must be considerably reduced to minimize global warming. Thus, the establishment of a consolidated production of polymers prioritizing the use of renewable sources of raw materials became a hot research topic. Vegetable oils are protagonists of this initiative, and their carbon-carbon double bonds are convenient reactive sites for chain growth polymerization reactions. However, typical vegetable oil-based homopolymers often do not display competitive thermomechanical properties, and the preparation of the corresponding copolymers and composites is therefore an interesting alternative strategy. Herein, the preparation of composites based on a tung oil/furfuryl alcohol
\end{abstract}

H. A. S. Valentino .

P. de Tarso Laia dos Reis e Silva Pupio .

T. M. Lacerda $(\bowtie)$

Biotechnology Department, Engineering School of

Lorena, University of São Paulo, Estrada Municipal do

Campinho, s/n - Campinho, Lorena,

São Paulo 12602-810, Brazil

e-mail: talitalacerda@usp.br

A. Gandini

LGP2, University Grenoble Alpes, 38000 Grenoble,

France co-continuous network reinforced with bacterial cellulose fibers is described. For this purpose, the cellulose nanofibers were suspended in furfuryl alcohol, and different amounts of the ensuing suspension were mixed with tung oil in the presence of trifluoroacetic acid as cationic initiator. Fourier-transform infrared spectroscopy analysis of all samples indicated the association of both tung oil and furfuryl alcohol in the final materials, with peaks belonging to cellulose superposed at the fingerprint regions of composites. Differential scanning calorimetry and thermogravimetry demonstrated an interesting relationship between the composition and the corresponding thermal properties, and the morphology of the materials was assessed by scanning electron microscopy (SEM), which revealed a homogeneous distribution of cellulosic fibers at lower concentrations. The results gathered here contribute to the development of original macromolecular materials exclusively based on the renewable platform.

Keywords Renewable composites - Vegetable oil · Cellulosic nanofibers $\cdot$ Solvent exchange $\cdot$ Furfuryl alcohol

\section{Introduction}

Vegetal and animal biomasses are very useful platforms for the development of polymeric materials. The 
consolidation of this strategy is now boosted by widespread discussions on how polymers should advance in a sustainable society based on a circular economy (Kawashima et al. 2019). Also, it contributes to a significant reduction in environmental impacts and opens the way for the preparation of materials with original microstructures and unprecedented properties. An efficient conversion of vegetable biomass into monomers and polymers, together with a correct and reduced disposal of wastes, have sparked the search for more sustainable polymeric materials (John et al. 2019; Gandini et al. 2016; Gandini and Lacerda, 2015). In fact, the large-scale production of biobased polymers using consolidated technologies, or those under advanced development, which is the case of biobased ethylene, ethylene glycol and terephthalic acid, are strong realities of such a trend (Hillmyer 2017).

In this context, vegetable oils are often considered as promising raw materials (Gandini and Lacerda 2019; Zhang et al. 2017) because of their high availability and of the long aliphatic skeleton that constitutes their molecules, which corresponds to the major element of the polymer chain to be formed. To pursue novel polymers from vegetable oils, different approaches are possible, including, among others, the breakdown of triglycerides by transesterification processes and the chemical modification of the fatty acids' double bonds by means of metathesis, epoxidation and click reactions. The various possibilities in this regard lead to the production of macromolecular materials with interesting properties and applications, such as thermosets, linear polyesters, polyurethanes, polyamides, and their blends and composites (Conti Silva et al. 2021; Quirino et al. 2021; Di Mauro et al. 2021; Mucci et al. 2020; Chakraborty and Chatterjee 2020; Lomège et al. 2019). Indeed, one can define some crude vegetable oils as natural oligomers, and film coatings based exclusively on unsaturated triglycerides have been used by man for millennia.

Tung oil is a vegetable oil which has as its major fatty acid $\alpha$-eleoestearic acid $((9 \mathrm{Z}, 11 \mathrm{E}, 13 \mathrm{E})$-octadeca-9,11,13-trienoic acid), bearing three conjugated double bonds (Fig. 1). In the last decades, due to its low price, large availability, and peculiar chemical structure, the intensive exploitation of tung oil in different contexts confirms its high potential to act as a rich source of molecules for the production of energy and materials. As a non-edible vegetable oil, it may be easily incorporated to the biodiesel industry without impacting the food and feed supply chain (Panchal et al. 2020).

The use of tung oil for the synthesis of polymers is, however, much more promising in terms of the possibilities to develop smart functional materials that can be useful for a wide domain of applications. The three conjugated double bonds of its main fatty acid allow the occurrence of oxidopolymerization, a reaction with atmospheric oxygen that naturally converts the triglyceride into a crosslinked polymer, which is particularly useful in the domain of paints and varnishes. The highly unsaturated backbone also makes it promptly reactive to chain-growth polymerizations (Sharma and Kundu 2006), as the occurrence of resonance stabilizes the intermediates involved in the process. The curing mechanism of tung oil under UV irradiation was investigated (Huang et al. 2018), which involved the contributions of both oxidative and free radical polymerization. Tung oil was also submitted to cationic polymerization reactions within a wide strategy involving its homopolymerization (Hondred et al. 2014a, b) and its association with other reactive counterparts for the synthesis of copolymers (Madbouly et al. 2014; Sibaja et al. 2014). In these and other cases reported in the literature, $\mathrm{BF}_{3}$ was used as initiator, in conditions often involving relatively high temperatures and oxidant atmosphere. More recently, the cationic polymerization mechanism of tung oil fatty acid methyl ester (methyl $\alpha$-eleostearate) initiated by a triarylsulfonium salt was investigated (Huang et al. 2019). Again, the authors reported the utilization of high temperatures and UV irradiation to achieve high conversions. The lack of available information and experimental evidences about the mechanisms involved in the cationic polymerization of tung oil motivated a systematic study, using trifluoroacetic acid as initiator under a nitrogen atmosphere (Lacerda and Gandini 2020).
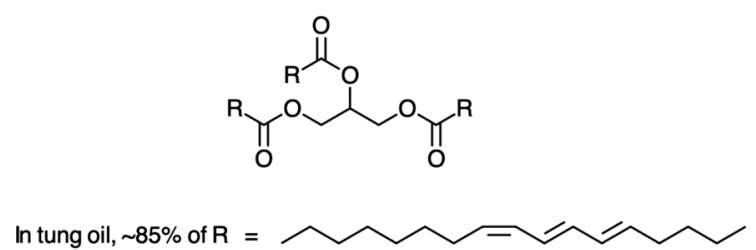

Fig. 1 Molecular structure of a generic triglyceride the main fatty acid found in the composition of tung oil 
The homopolymerization of tung oil leads to highly flexible materials with limited performance, and the use of comonomers can provide improved stiffness to the final polymers. Styrene and divinylbenzene are often the choice in this case (Meiorin et al. 2015; Hondred et al. 2014a, b), giving materials with broader potential applications. Similar effects can be achieved by using furan derivatives as comonomers, since furfuryl alcohol and furan resins can be polymerized by acid catalysis to provide infusible solid products with high hardness, flexural strength, and chemical resistance. Recently, the copolymerization reaction of crude tung oil and furfuryl methacrylate initiated by benzoyl peroxide under an inert atmosphere was described, and the final networks were obtained in a second step based on the unusual Diels-Alder click reaction between pendant furan moieties and residual conjugated double bonds of tung oil, which was performed under high pressure and high temperature (Sain et al. 2020a). The same authors applied the ensuing networks as matrixes to produce biocomposites reinforced with onion-skin-extracted cellulose nanofibers and cellulose nanocrystals (Sain et al. 2020b).

In fact, the possibility of using natural fibers as reinforcing agents in composites with matrices also based on renewable sources is a strategy that leads to materials totally independent of the petrochemical platform. O'Donnell et al. (2004) described the preparation of composites of acrylated epoxidized soybean oil-based polymers reinforced with cellulose fibers of different origins. Quirino and Larock (2009; 2011a; 2011b) prepared composited with a matrix obtained by the free radical polymerization of a mixture of conjugated soybean oil, divinylbenzene, n-butyl methacrylate and dicyclopentadiene with soybean hulls as reinforcement. The same authors produced similar composites based on different vegetable oils (soybean, flaxseed and tung oils), reinforced with sugarcane bagasse fibers (Quirino and Larock 2012). Yoo and Youngblood (2017) reported the preparation of cellulose nanocrystal (CNC)-reinforced tung oil composites as an effective option for wood finishes that offers enhanced mechanical and weathering performance owing to the high strength, stiffness, and barrier properties of CNC (Clarkson et al. 2020). A heat treatment was performed on tung oil at $100-200{ }^{\circ} \mathrm{C}$ to modify bamboo materials, and the synergistic effects of tung oil and heat treatment on the chemical, physical and mechanical properties of these composites were investigated (Tang et al. 2019). Results indicated that the ensuing materials had enhanced hydrophobic properties, dimensional stability and fungi resistance, as well as good mechanical performances.

To the best of our knowledge, only one study was reported on the preparation of vegetable oil-based matrices via cationic polymerization, with natural fibers (corn cob, wheat straw and Panicum virgatum grass) as reinforcing agents (Pfister and Larock 2012). Materials bearing $75 \mathrm{wt} \%$ of natural fibers displayed high thermal stability, and Young modulus and tensile strength up to $2300 \mathrm{MPa}$ and $11.3 \mathrm{MPa}$, respectively.

Considering our previous investigation on the cationic copolymerization of tung oil and furfuryl alcohol using trifluoroacetic acid as initiator (Ribeiro et al. 2020), and the entirely renewable origin of the ensuing materials, its association with natural fibers to produce composites has potential to widen the scope of possible applications. Herein, a fully renewable composite material based on the cationic polymerization of tung oil and furfuryl alcohol in the presence of bacterial cellulose is investigated with the aim of chemically combining hard and soft networks incorporating a stiffening nanofiber (Gallegos et al. 2016) and thus being able to modulating its properties as a function of its composition. Given the rapid homopolymerization of furfuryl alcohol in acid conditions (Choura et al. 1996), trifluoroacetic acid (TFA), an acid of moderate strength $(\mathrm{pKa}=0.23$ ), was used as cationic initiator. For this purpose, bacterial cellulose nanofibers were suspended for the first time in furfuryl alcohol, which is a very interesting strategy in this case considering (1) the incompatibility of water with the cationic mechanism, (2) the complete miscibility of water and furfuryl alcohol in all proportions, which avoids the need of an intermediate solvent for drying the nanofibers, and (3) the fact that furfuryl alcohol itself is one of the two comonomers involved in the synthesis of the copolymer matrix. Also, as recently reported, the preparation of composites based on tung oil and cellulose fibers, which respectively correspond to continuous and discontinuous phases of very different polarities, must be carried out in such a way as to favor the matrixreinforcement compatibility, leading to materials of improved properties (Murawski and Quirino 2019). Therefore, considering the polymerization mechanism 
and the final structures of the copolymers that constitute the continuous phase of the composites prepared here (Ribeiro et al. 2020), furfuryl alcohol could act as an additional compatibilization agent in this case (Fig. 2), since the presence of ester groups in general triglycerides may intervene synergically. The development of these original materials may contribute to the advancement of fully renewable materials with tunable properties.

\section{Experimental}

\section{Materials}

Tung oil (TO) was purchased from General Iron Fittings Ltda. (São Paulo, Brazil). Furfuryl alcohol (FA, Sigma-Aldrich, 98\%), 1,5,7-triazabicyclo[4.4.0]dec-5-ene (TBD, Sigma-Aldrich, 98\%), chloroform (Qhemis, 99\%), dichloromethane (Qhemis, 99\%), triethylamine (Sigma-Aldrich, $\geq 99 \%$ ) and microcrystalline cellulose (Sigma-Aldrich) were used as received unless otherwise stated. Trifluoroacetic acid (TFA, Sigma-Aldrich, 99\%) was used in a chloroform solution. Bacterial cellulose (BC) nanofibers were obtained from kombucha tea, which is a symbiotic culture of bacteria and yeasts.

\section{Instrumentation}

Fourier-transform infrared (FTIR) spectra were acquired in a PerkinElmer Spectrum 100, in attenuated total reflection (ATR) mode. The thermal properties were assessed by thermogravimetric analysis (TGA) with a Netzsch STA instrument, operating from 25 to $700{ }^{\circ} \mathrm{C}$ at $10{ }^{\circ} \mathrm{C} \mathrm{min}^{-1}$, working with a nitrogen flow of $20 \mathrm{~mL} \mathrm{~min}^{-1}$ and temperature ranging from 25 to $700{ }^{\circ} \mathrm{C}$, with a heating rate of $10{ }^{\circ} \mathrm{C} \mathrm{min}^{-1}$. Differential Scanning Calorimetry (DSC) analysis were conducted using a TA Q10 instrument with temperature ranging from -70 to $250{ }^{\circ} \mathrm{C}$ with a heating rate of $10{ }^{\circ} \mathrm{C} \mathrm{min}^{-1}$. SEM micrographs were acquired in a Field Emission Scanning Electron Microscope (FESEM) Jeol JSM-7401F, using gold-coated samples under varying magnifications.
Preparation of bacterial cellulose/furfuryl alcohol suspension

Solvent exchange for the removal of water is often reported in the context of nanofibrillated cellulose surface modification, including the use of toluene, $N, N$-dimethylformamide (DMF), dimethylacetamide (DMA), ionic liquids, among others (Missoum et al. 2013). However, the suspension of bacterial cellulose (BC) in furfuryl alcohol corresponds to a strategy that was not previously reported in the literature. BC was obtained from kombucha tea culture (Dufresne and Farnworth 2000). As culture medium, a 1 L solution containing $20 \mathrm{~g}$ of sucrose, $20 \mathrm{~mL}$ of commercial vinegar (nominal acetic acid concentration of $5 \% \mathrm{v} / \mathrm{v}$ ), and commercial black tea extract $(20 \mathrm{~g})$ was used. A membrane containing the cellulose producing microorganisms was immersed in the culture medium, and static fermentation was conducted for approximately 7 days, when it was possible to observe the presence of a floating thick cellulosic membrane on the surface. The membrane was purified in a $0.1 \mathrm{M}$ $\mathrm{NaOH}$ solution, at $60{ }^{\circ} \mathrm{C}$, for $30-60 \mathrm{~min}$, washed with distillated water until constant $\mathrm{pH}$, and the fibers were dispersed in water with an UltraTurrax apparatus (IKA T25), giving a suspension of approximately $1 \mathrm{wt} \%$. The cellulosic nature of the material was confirmed by comparing the FTIR spectra of the dried nanofibers with commercial microcrystalline cellulose (Avicel), using a Perkin Elmer spectrometer (Spectrum GX), working in a transmission mode. Sample pellets were prepared with $\mathrm{KBr}$ (spectroscopic grade), kept in an oven at $105{ }^{\circ} \mathrm{C}$ for $60 \mathrm{~min}$. For solvent exchange of cellulose suspensions, sequences of centrifugation, removal of the supernatant, addition of furfuryl alcohol and re-suspension of fibers were conducted (Johansson et al. 2011). Since water and furfuryl alcohol are miscible in all proportions, the use of an intermediate solvent (such as acetone) was not necessary. These steps were repeated ten times to assure the total removal of moisture.

Synthesis of copolymers and composites based on tung oil and furfuryl alcohol

A preliminary experiment was conducted to select a TFA concentration that was sufficient to promote gel formation in reasonable time lengths, considering the two-step synthesis of the copolymer. $2 \mathrm{~g}$ of crude tung 


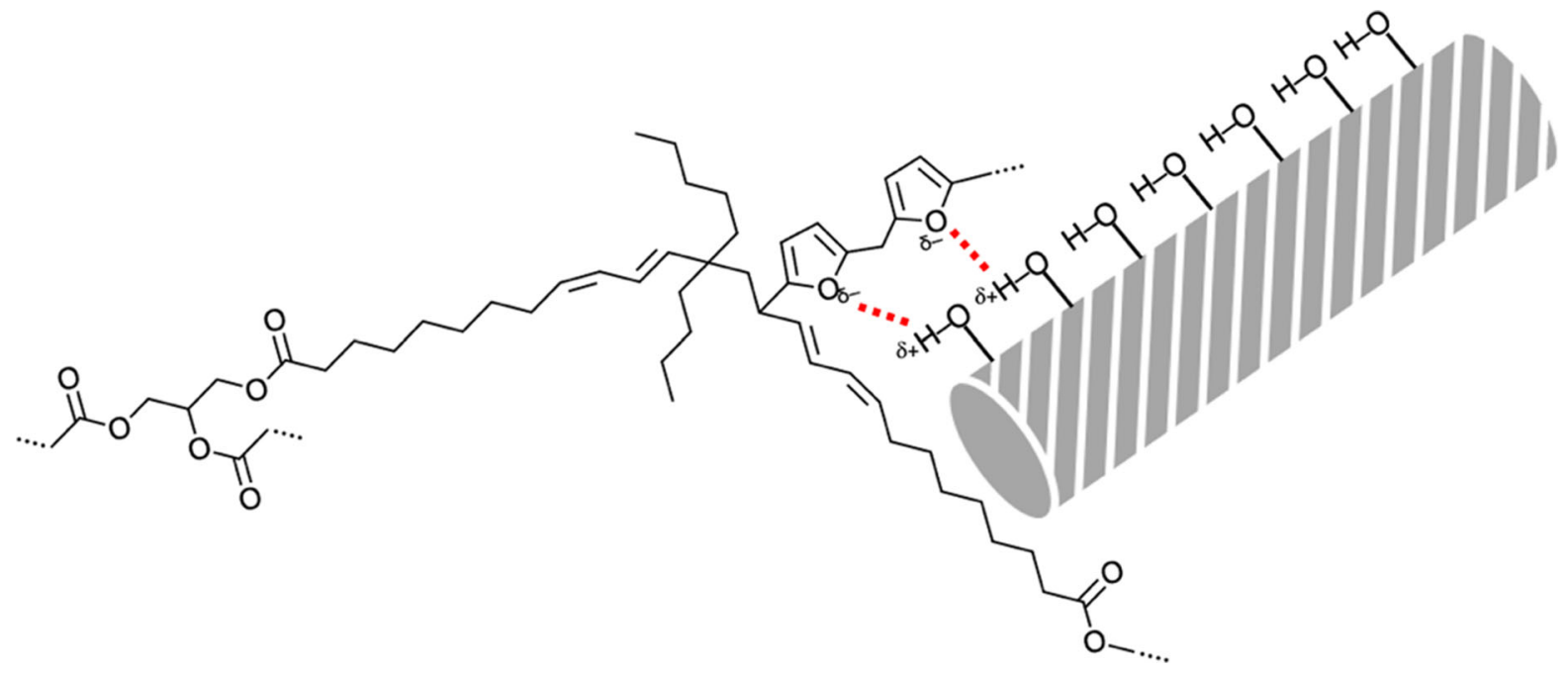

Fig. 2 Schematic representation of the action of furfuryl alcohol favoring the interaction between continuous and disperse phases

oil were mixed with $2 \mathrm{~mL}$ of a TFA solution in chloroform, to give final concentrations of $0.5,0.6,0.7$ and $0.8 \mathrm{M}$ of TFA. Gel formation was observed, respectively, after approximately $30 \mathrm{~h}, 7 \mathrm{~h}, 1 \mathrm{~h}$ and $0.5 \mathrm{~h}$. The concentration of $0.5 \mathrm{M}$ of TFA was then selected to proceed with the synthesis of copolymers (CP) with furfuryl alcohol, and the specific formulation used in each reaction is given in Table 1. The composites (CPST) were prepared following the same procedure.

In all cases, the flasks were kept at room temperature under a nitrogen atmosphere, left under magnetic stirring for $5 \mathrm{~min}$ at the beginning of the reaction and after furfuryl alcohol (or furfuryl alcohol/BC suspension) addition, and left to rest until gelation was observed. The gels were then re-suspended in chloroform, and the acid was neutralized with triethylamine under vigorous magnetic stirring. The choice of triethylamine was made based on its ability to efficiently neutralize acids of moderate strength, such as trifluoroacetic acid and acetic acid (Barrow and Yerger 1954), in non-dissociating solvents, forming a stable ion-pair. The insoluble fraction was isolated by filtration and extracted with dichloromethane in a Soxhlet system to remove unreacted monomers, soluble oligomers, and triethylammonium salts. Both copolymers (CP1-CP3) and composites (CPST1CPST8) were characterized by infrared spectroscopy (FTIR/ATR), thermal analysis (TGA and DSC) and scanning electron microscopy (FEG/SEM).

\section{Results and discussion}

Figure 3 shows the FTIR spectra and SEM micrograph of isolated bacterial cellulose fibers. For the FTIR analysis, commercial microcrystalline cellulose was used for comparison purposes. There is a coincidence between the bands presented in the two cases (Fig. 3 a), which appear at the same wavenumbers but with different relative intensities. Typical cellulose bands are found at (1) $3000-3600 \mathrm{~cm}^{-1}$, from the $\mathrm{O}-\mathrm{H}$ stretch of glucopyranose hydroxyls; (2) $2900 \mathrm{~cm}^{-1}$, from $\mathrm{C}-\mathrm{H}$ bonds stretching; (3) between 1050 and $1100 \mathrm{~cm}^{-1}$, associated with vibrations of $\mathrm{C}-\mathrm{O}$ bonds; and (4) around $700 \mathrm{~cm}^{-1}$, related to vibrations of $\mathrm{C}-\mathrm{H}$ and $\mathrm{H}-\mathrm{C}-\mathrm{H}$ bonds. In contrast to microcrystalline cellulose, the relative intensity of the band corresponding to hydroxyl group in the region of $4000-3000 \mathrm{~cm}^{-1}$ was lower with respect to the $\mathrm{C}-\mathrm{H}$ bond stretching at $2900 \mathrm{~cm}^{-1}$ for the spectra of bacterial cellulose fibers, which may be due to the higher number of hydroxyl groups participating in strong hydrogen bonds in the bacterial cellulose sample (El-Saied et al. 2008; Levdik et al. 1967). The main peaks between 2000 and $500 \mathrm{~cm}^{-1}$ are related to the axial disposition of the anomeric hydrogen atom in B-glucopyranose unit, which leads to Van der Waals interaction with the axial hydrogen on $\mathrm{C}_{5}$, increasing the frequency of the deformation vibration involving $\mathrm{C}_{1}-\mathrm{H}$ (Higgins et al. 1961). Also, it is important to mention the presence of a band in $1723 \mathrm{~cm}^{-1}$, from ester carbonyls, in the sample of 
Table 1 Formulations used for the preparation of copolymers (CP) and composites (CPST) based on tung oil and furfuryl alcohol

*Added approximately $4 \mathrm{~h}$ reaction; ** added approximately $2 \mathrm{~h}$ after the beginning of the reaction. In all cases, the final concentration of TFA was $0.5 \mathrm{~mol} \mathrm{~L}^{-1}$ after the beginning of the

\begin{tabular}{llll}
\hline Sample & Tung oil $(\mathrm{mL})$ & Furfuryl alcohol $(\mathrm{mL})$ & Furfuryl alcohol/BC suspension $(\mathrm{mL})$ \\
\hline CP1 & 0.90 & $0.10^{*}$ & - \\
CP2 & 0.95 & $0.05^{*}$ & - \\
CP3 & 0.98 & $0.02^{*}$ & - \\
CPST1 & 0.90 & - & $0.10^{*}$ \\
CPST2 & 0.95 & - & $0.05^{*}$ \\
CPST3 & 0.98 & - & $0.02^{*}$ \\
CPST4 & 0.50 & - & $0.50^{* *}$ \\
CPST5 & 0.40 & - & $0.60^{* *}$ \\
CPST6 & 0.30 & - & $0.70^{* *}$ \\
CPST7 & 0.20 & - & $0.80^{* *}$ \\
CPST8 & 0.10 & - & $0.90^{* *}$ \\
\hline
\end{tabular}

bacterial cellulose, which may be due to contamination of cells or protein residues (Fuller et al. 2018). It is possible that even after the purification step, there were still cells (or cell debris) left in the membranes. The morphology of the nanofibers (Fig. 3b) is in accordance with other SEM micrographs of bacterial cellulose reported in the literature (Gandini et al. 2016; Gomes et al. 2013), with fibrils of long length and nanometric diameter. Typically, the diameter of bacterial cellulose fibers ranges from 20 to $100 \mathrm{~nm}$ (Manoukian et al. 2019), giving their well-known high aspect ratio.

A thorough characterization of tung oil, furfuryl alcohol and their ensuing homopolymers was provided elsewhere (Lacerda and Gandini 2020; Ribeiro et al. 2020). The FTIR spectra of the copolymers (CP1, CP2 and CP3) and composites (CPST1-CPST8) (Table 1) prepared here are given, respectively, in Figs. 4 and 5, emphasizing wavenumber intervals of $4000-2500 \mathrm{~cm}^{-1}$ and 2000-650 $\mathrm{cm}^{-1}$.

The FTIR spectra of all samples tested displayed a similar profile, and it is possible to identify the presence of peaks coming from tung oil and furfuryl alcohol individual homopolymers in all samples, which indicates the association of both monomers in the final material, despite the low concentrations of furfuryl alcohol. Tung oil typically present peaks $\left(\mathrm{cm}^{-1}\right)$ at 3014,1640 , and 991, which correspond, respectively, to $s p^{2} \mathrm{C}-\mathrm{H}$ stretch, $\mathrm{C}=\mathrm{C}$ stretch and $=\mathrm{C}-$ $\mathrm{H}$ out of plane bending of the three conjugated unsaturations of the fatty acid (Lacerda and Gandini 2020). The furfuryl alcohol-based resins, on the other hand, show peaks at $3450 \mathrm{~cm}^{-1}$, from the $-\mathrm{OH}$ stretch, $2980 \mathrm{~cm}^{-1}$, which suggests the presence of methyl
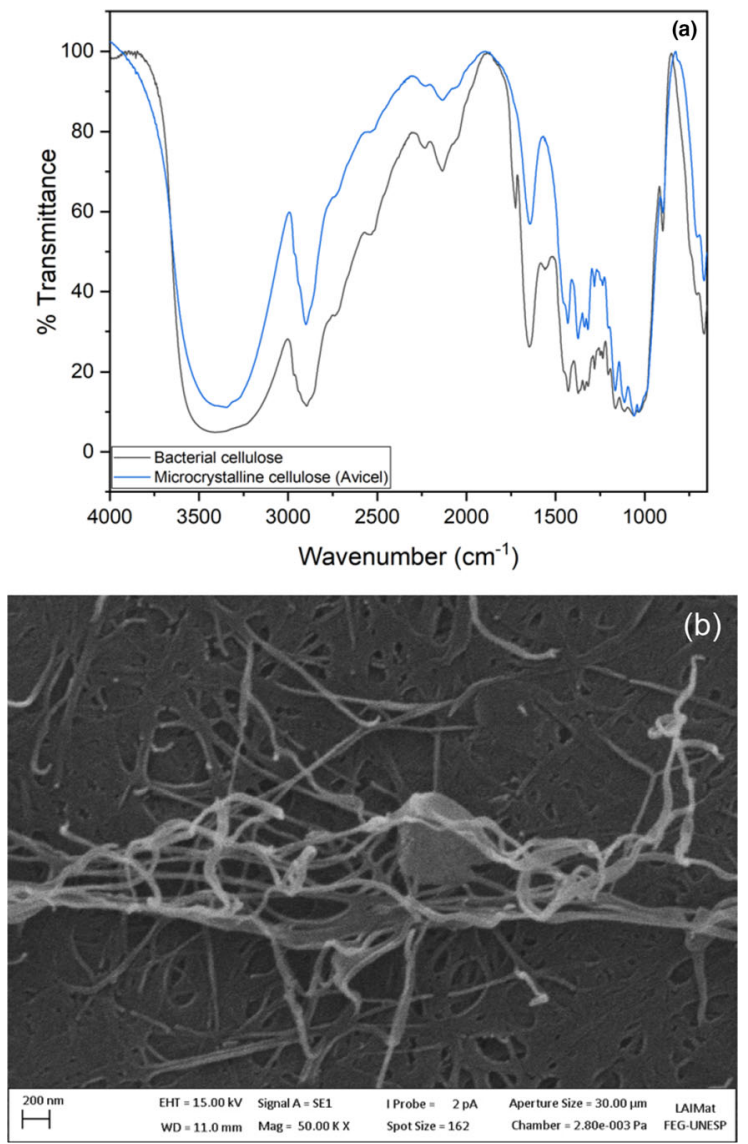

Fig. 3 a FTIR spectra of purified bacterial cellulose (black line) and microcrystalline cellulose (blue line); b SEM micrograph of purified bacterial cellulose nanofibers

groups, $1715 \mathrm{~cm}^{-1}$, typical of carbonyl groups, suggesting the occurrence of some ring-opening induced by the acidic medium in the presence of the 
accumulated condensation water, and $793 \mathrm{~cm}^{-1}$, characteristic of 2,5-disubstituted furan rings (Choura et al. 1996). It is important to mention that, as previously indicated, the polymerization was initiated with tung oil alone to favor the formation of tung oil oligomers prior to the addition of furfuryl alcohol. The absence of a band at around $3014 \mathrm{~cm}^{-1}$, from C $=\mathrm{C}-\mathrm{H}$ stretching vibrations, suggests a high consumption of the double bonds through the incorporation of tung oil monomers into the copolymer chains (Lacerda and Gandini 2020; Ribeiro et al. 2020).

Composites CPST1-CPST8 were prepared with different contents of bacterial cellulose nanofibers suspended in furfuryl alcohol with respect to tung oil (Table 1). The presence of peaks coming from tung oil and furfuryl alcohol polymers in all samples was again observed. In the spectra of samples CPST1-CPST3, prepared with lower concentrations of furfuryl alcohol/bacterial cellulose, it was not possible to identify bands attributable to cellulose chains. When higher concentrations of the suspension were however employed (CPST4-CPST8), the contribution of bands from the segments of furfuryl alcohol resin became more significant (Ribeiro et al. 2020), leading to bands superposition at the fingerprint regions, thus covering the peaks belonging to cellulose.

Figures 6 and 7 show the results of DSC and TGA analyses, separately for copolymers (CP1-CP3) and composites (CPST1-CPST8). All DSC measurements were conducted with two cycles of heating/cooling, with temperatures ranging from -70 to $250{ }^{\circ} \mathrm{C}$ at a

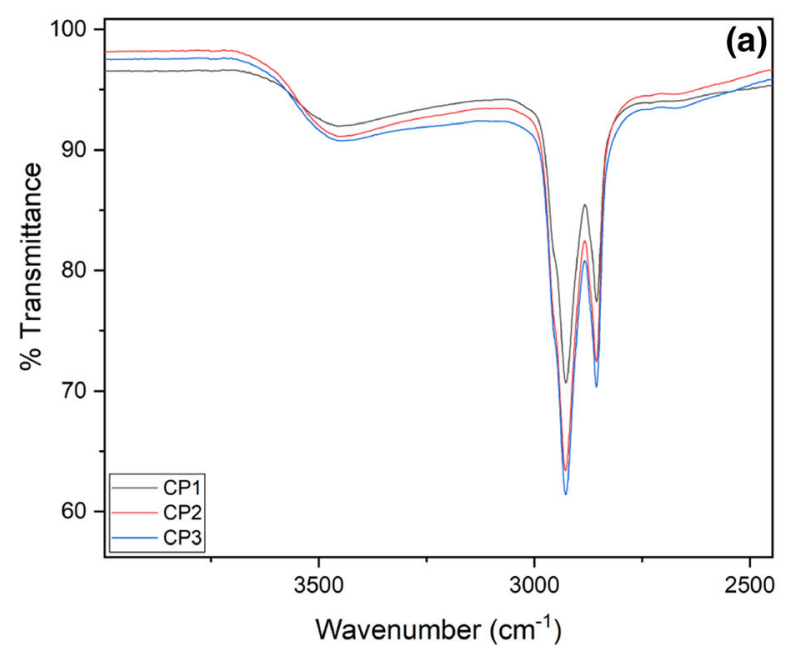

rate of $10{ }^{\circ} \mathrm{C} \min ^{-1}$, and the DSC curves shown here correspond to the second heating in all instances.

The three samples in Fig. 6 a exhibited a small endothermic peak near $0{ }^{\circ} \mathrm{C}$, which was attributed to the melting of ice formed during cooling on the first heating/cooling cycle and arising from the water produced during the furfuryl alcohol polycondensation (Choura et al. 1996). Assuming that the experimental conditions involve relatively fast heating and cooling rates $\left(10^{\circ} \mathrm{C} \mathrm{min}^{-1}\right)$, it is possible that residual water molecules do not have enough time to diffuse and remain trapped into the gel, appearing on the second heating as well. This peak could have masked the glass transition, expected at values near $0{ }^{\circ} \mathrm{C}$, reported for the tung oil homopolymer (Lacerda and Gandini 2020).

The mass loss behavior of the copolymers displayed in Fig. $6 \mathrm{~b}$ are in accordance with results previously reported for tung oil-based homopolymer (Lacerda and Gandini 2020) and its copolymers with furfuryl alcohol (Ribeiro et al. 2020), with the samples exhibiting thermal stability up to $200{ }^{\circ} \mathrm{C}$ and approximately $30 \%$ weight loss recorded at around $400{ }^{\circ} \mathrm{C}$. The thermograms showed the minor influence of furfuryl alcohol in these cases, which was expected considering its low concentration used in the formulations, with graphitic residues of between 5 and $10 \%$ of the initial mass at $700{ }^{\circ} \mathrm{C}$.

The DSC curves in Fig. 7 a, in the case of the composites CPST1-CPST3, bearing lower amounts of furfuryl alcohol, displayed glass transitions, which

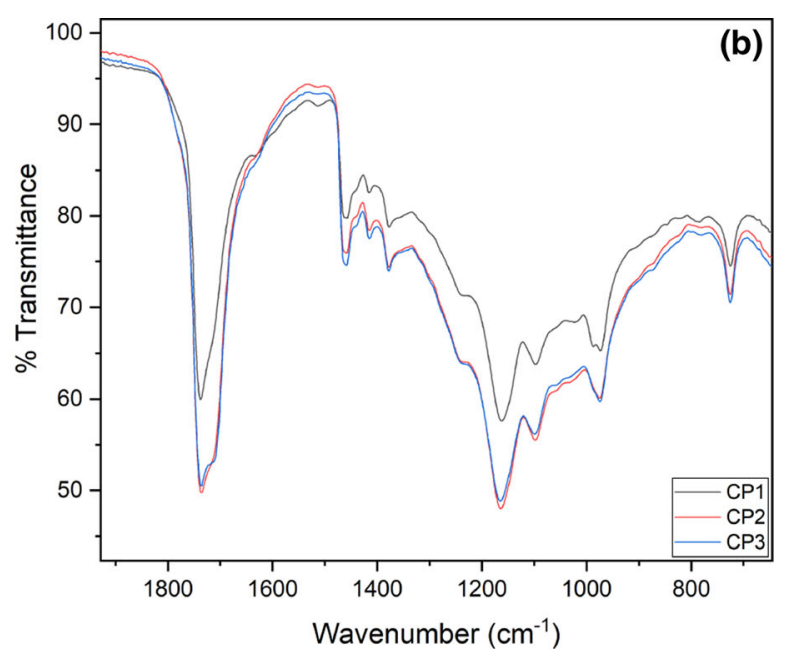

Fig. 4 FTIR spectra of samples CP1, CP2 and CP3, at a 4000-2500 $\mathrm{cm}^{-1}$ and b $2000-650 \mathrm{~cm}^{-1}$ 
(a)

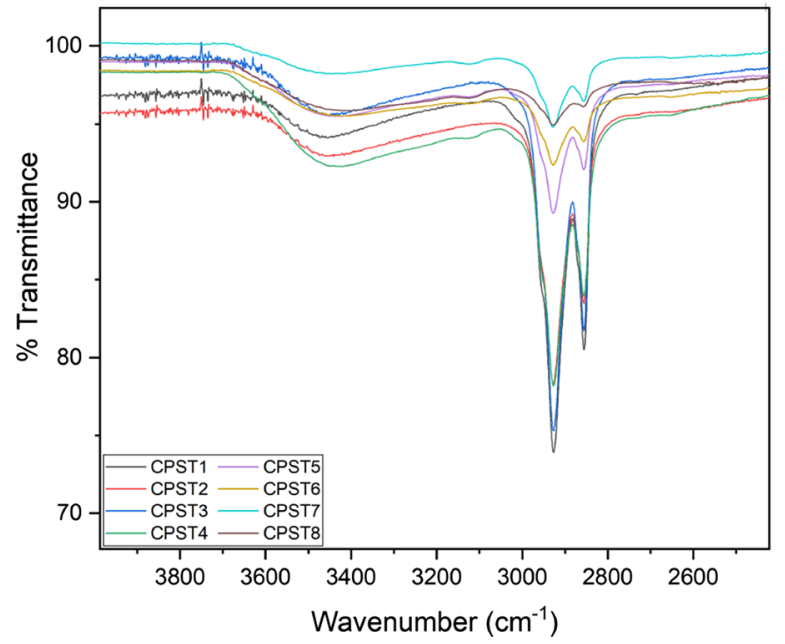

(b)

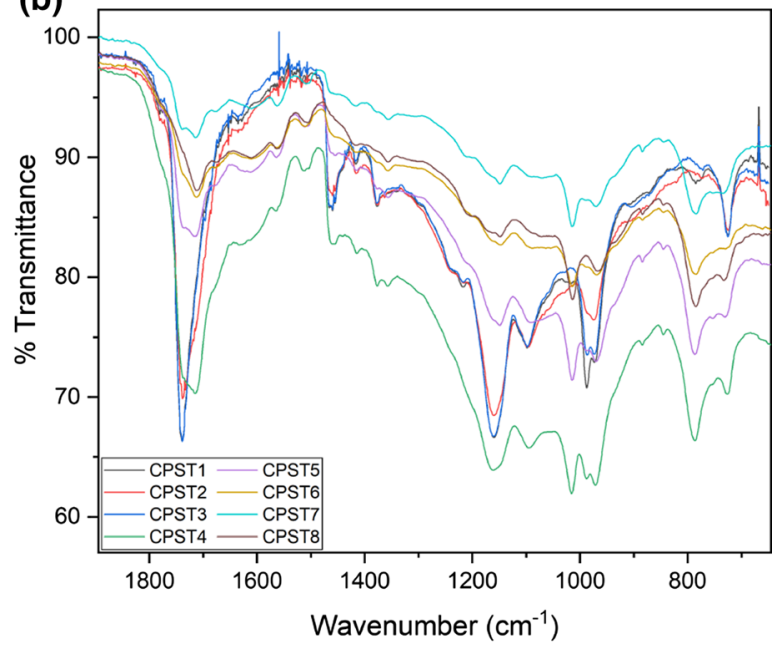

Fig. 5 FTIR spectra of samples CPST1-CPST8, at a 4000-2500 $\mathrm{cm}^{-1}$ and b 2000-650 cm $\mathrm{cm}^{-1}$

increased moderately from 2 to about $12{ }^{\circ} \mathrm{C}$, from the $0{ }^{\circ} \mathrm{C}$ of the neat tung oil network (Lacerda and Gandini 2020), thus reflecting the growing, albeit modest, stiffening effect of the incorporation of furan moieties in the co-network. This result was expected considering the concentrations of bacterial cellulose/furfuryl alcohol suspension tested, that were not high enough to provide a strong increase in the rigidity to the final materials. The composites CPST4-CPST8, bearing progressively higher amounts of furfuryl alcohol, exhibited again the melting ice endothermic peak near $0{ }^{\circ} \mathrm{C}$, whose magnitude increased, as expected, as this amount was increased because of the correspondingly higher amount of condensation water released. In the second part of these curves, broad glass transitions appeared, which increased from about $60{ }^{\circ} \mathrm{C}$ for the lower, to $105{ }^{\circ} \mathrm{C}$ for the highest amount of bacterial cellulose/furfuryl alcohol used in the original compositions with tung oil, reflecting the expected more substantial stiffening effect brought about by progressively higher presence of both the furan polymer in the co-network and the nanocellulose content. Interestingly, all these DSC tracings displayed a single glass transition which varied as a function of tung oil-furfuryl alcohol composition, strongly suggesting that the two
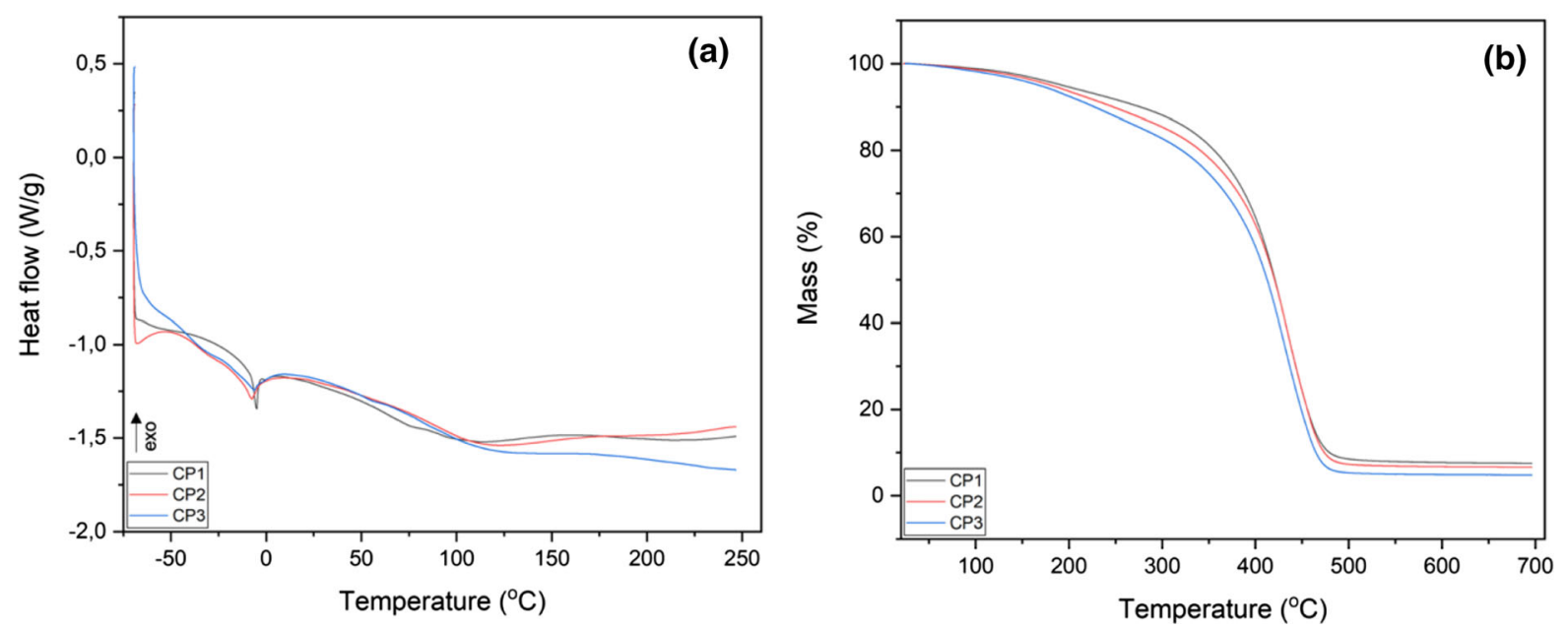

Fig. 6 a DSC traces and b TGA curves of samples CP1, CP2 and CP3 


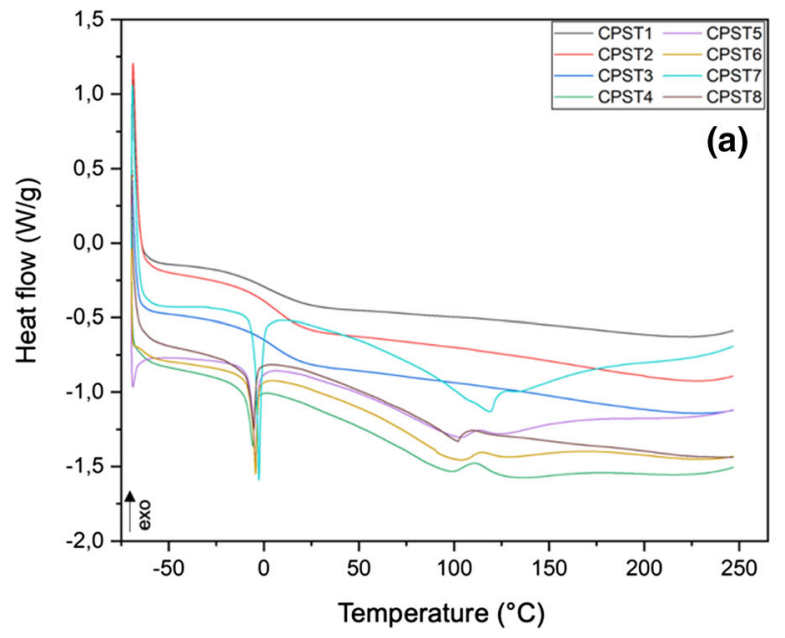

Fig. 7 a DSC traces and b TGA curves of samples CPST1-CPST8

monomers had generated crosslinked materials in which the corresponding polymers were linked covalently in a single three-dimensional architecture. This important conclusion was confirmed by the SEM results discussed below.

The mass loss behavior of the composites (Fig. 7b) can be separated into two very distinct situations, considering the concentrations of bacterial cellulose fibers suspended in furfuryl alcohol with respect to tung oil. The composites prepared with lower suspension contents (CPST1-CPST3) gave thermograms very similar to those of the analogous copolymers (CP1-CP3, Fig. 4b). Again, for these three samples, thermal analysis indicated a small influence of furfuryl alcohol, with graphitic residues of $5-10 \%$ of the initial mass were recorded at $700{ }^{\circ} \mathrm{C}$. On the other hand, the other set of copolymers prepared with higher amounts of the bacterial cellulose/furfuryl alcohol suspension (CPST4-CPST8) exhibited an interesting pattern that was directly related to the contribution of the furfuryl alcohol resin, as already observed in a previous study on the tung oil-furfuryl alcohol cationic copolymerization [29]. All these composite samples exhibited thermal stability up to about $300{ }^{\circ} \mathrm{C}$ and, again, approximately $30 \%$ weight loss at around $400{ }^{\circ} \mathrm{C}$. The graphitic residues at $700{ }^{\circ} \mathrm{C}$ were however considerably higher here and indeed the higher, the higher the content of furfuryl alcohol used in the composite composition, namely $30.9 \%, 34.4 \%, 36.5 \%, 39.1 \%$ and $51.3 \%$, respectively for samples CPST4, CPST5, CPST6, CPST7 and CPST8, confirming the growing

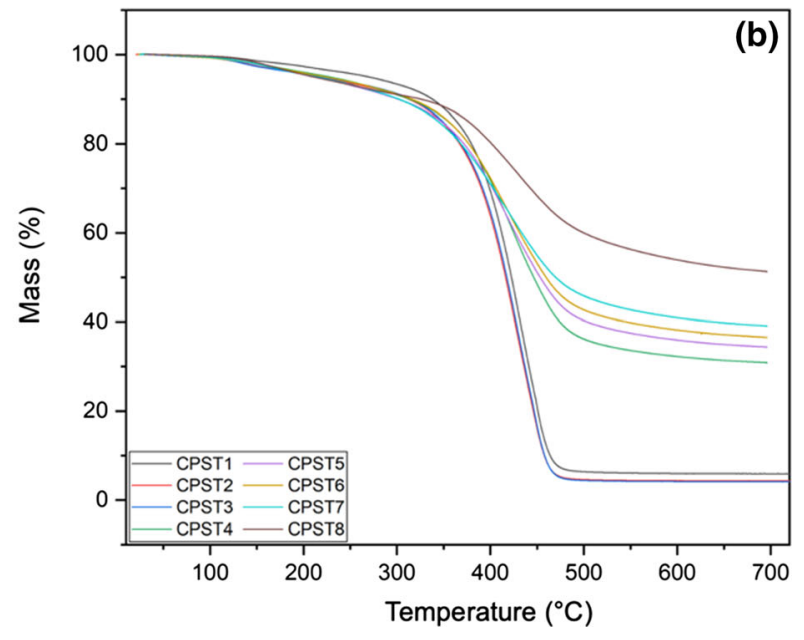

presence of the furan polymer in the final materials [29].

The morphology of all samples was assessed by SEM, and representative micrographs are shown in Figs. 6 and 7.

The micrographs in Figs. $8 \mathrm{a}, \mathrm{b}$ and c correspond to the unreinforced copolymers $\mathrm{CP} 1, \mathrm{CP} 2$ and $\mathrm{CP}$, respectively, whereas those in Figs. 8d-f refer to composites with lower bacterial cellulose fibers/furfuryl alcohol content CPST1, CPST2 and CPST3, respectively. The copolymers $\mathrm{CP} 1, \mathrm{CP} 2$ and $\mathrm{CP} 3$ exhibited a compact, homogeneous and dense morphology, which indicated the formation of a continuous co-network, with no phase separation. The presence of some micrometric fragments on the materials surface was also observed (as highlighted with a black narrow in Fig. 8a). EDS analysis did not show any difference in the chemical compositions of the fragments. It was already possible to observe the presence of fibers in samples CPST2 and CPST3 (Figs. 8e and f highlighted with black arrows) with diameters in accordance with bacterial cellulose morphology [36]. Despite the difference in polarity between cellulose and plant oil-based polymers, the adhesion of the continuous phase to the surface of cellulose was clearly established, most probably thanks to the initial fiber impregnation with furfuryl alcohol and to the presence of its polymer, despite its low concentration.

Figure 9 shows surface micrographs of composites CPST4-CPST8. In all cases, it was possible to observe 

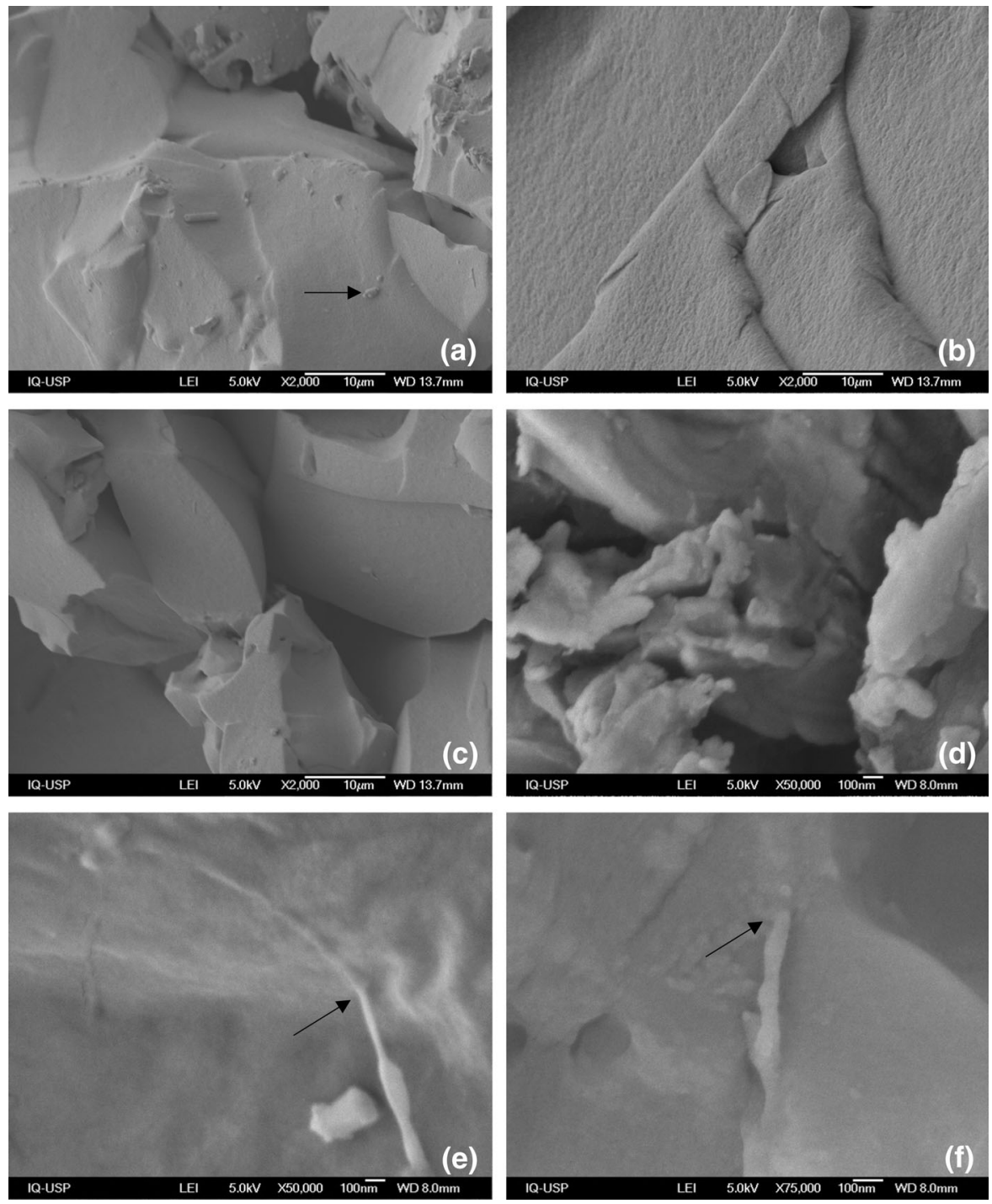

Fig. 8 SEM micrographs of a CP1, b CP2 and c CP3, at 2000×magnification; d CPST1, e CPST2 and f CPST3, at $50,000 \times$ magnification

the presence of a continuous compact matrix together with regions containing cellulosic fibers with a rather low degree of dispersion. This phenomenon was more striking for samples containing higher amounts of bacterial cellulose/furfuryl alcohol suspension, which can be due, on the one hand, to the known tendency of self-assembling of nanocellulose fibers, mainly when high concentrations are involved, and on the other hand, to the fact that higher contents of furfuryl alcohol favored its fast resinification, which hindered a homogeneous dispersion of the nanofibers in the final materials. This non-uniformity of the samples, consisting of a nanoccelulose-rich part and a resin-rich part was previously reported for similar materials [21], and may negatively impact the mechanical performance of the composites. However, it is also worth reemphasizing the good matrix-fibers compatibility, as indicated by the high adhesion between the phases. 
Fig. 9 SEM micrographs of CPST4 (a, b), CPST5 (c, d), CPST6 (e, f), CPST7 (g, h) and CPST $8(\mathbf{i}, \mathbf{j})$, at $5,000 \times$ (left column) and $50,000 \times$ (right column) magnification
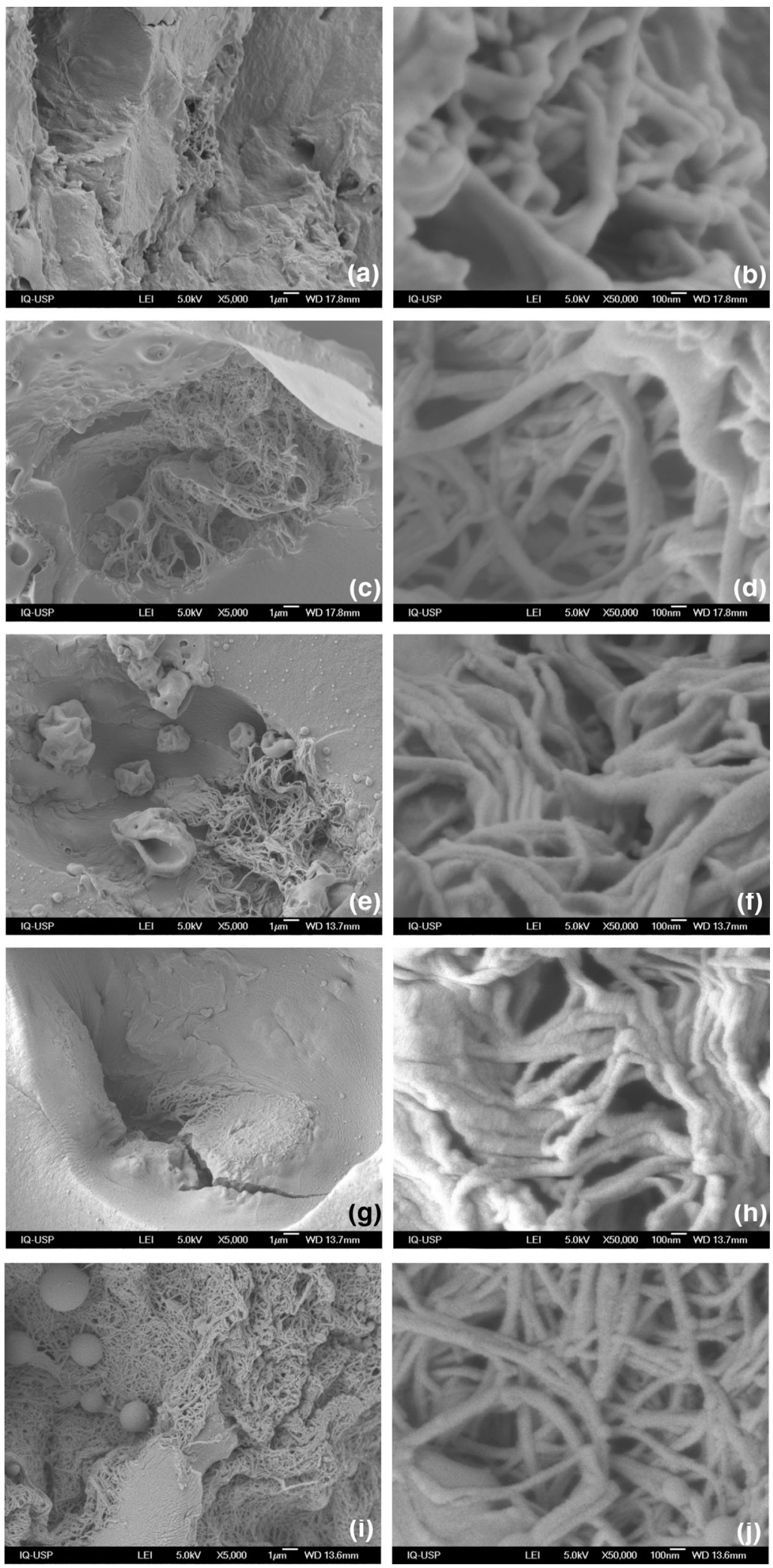


\section{Conclusions}

This study contributed with the development and manufacturing of fully bio-based composites based on a continuous phase of tung oil and furfuryl alcohol network and a disperse phase comprising cellulosic fibers. These novel composite materials were, therefore, successfully developed involving three very different building blocks bearing the important commonality of vegetable biomass origin and thus carrying an important sustainability connotation. For this purpose, bacterial cellulose fibers were dispersed, for the first time, in furfuryl alcohol, which paved the way to an in-situ building of the final materials with varying compositions. The results demonstrated the feasibility of such approach, and the characterization by spectroscopic, thermal, and surface morphology inspections provided evidence for the formation of a covalently bound co-network matrix and for full compatibility between matrix and filler, as well as for the possibility of modulating their properties as a function of the soft/hard component content. These encouraging results are however preliminary, and the optimized conditions needed to achieve an improved dispersion of the nanofibers, as well as the assessment of the ensuing mechanical properties are in progress, regrettably slowed down considerably by the constraints imposed by the Covid-19 pandemic.

Author contributions HASV-Methodology, validation, investigation. PTLRSP-methodology, validation, investigation. AG: conceptualization, methodology, writingreview and editing. TML: conceptualization, methodology, resources, writing — original draft, writing—review and editing, supervision, funding acquisition.

Funding This work was supported by FAPESP (São Paulo Research Foundation, Grant 2017/16062-6) and CNPq (National Council for Scientific and Technological Development, Grant 420023/2018-6).

Data availability University of São Paulo offers a scientific data repository, in which all the data related to the present manuscript may be uploaded.

\section{Declarations}

Conflict of interest The authors declare that they have no conflict of interest.

Consent to participate All the authors are aware and consented to participate as co-author in the present manuscript.
Consent to publication All the authors are aware and consented to publish the results presented in the document.

\section{References}

Barrow GM, Yerger EA (1954) Acid-base reactions in nondissociating solvents: acetic acid and triethylamine in carbon tetrachloride and chloroform. J Am Chem Soc 76:5211-5216

Chakraborty I, Chatterjee K (2020) Polymers and composites derived from castor oil as sustainable materials and degradable biomaterials: current status and emerging trends. Biomacromol 21:4639-4662

Choura M, Belgacem MN, Gandini A (1996) Acid-catalyzed polycondensation of furfuryl alcohol: mechanisms of chromophore formation and cross-linking. Macromolecules 29:3839-3850

Clarkson CM, El Awad Azrak SM, Forti ES, Schueneman GT, Moon RJ, Youngblood JP (2020) Recent developments in cellulose nanomaterial composites. Adv Mater 21:2000718

Conti Silva JA, Grilo LM, Gandini A, Lacerda TM (2021) The prospering of macromolecular materials based on plant oils within the blooming field of polymers from renewable resources. Polymers. (in press)

Di Mauro C, Genua A, Rymarczyk M, Dobbels C, Malburet S, Graillot A, Mija A (2021) Chemical and mechanical reprocessed resins and bio-composites based on five epoxidized vegetable oils thermosets reinforced with flax fibers or PLA woven. Compos Sci Technol. 205:108678

Dufresne C, Farnworth E (2000) Tea, Kombucha, and health: a review. Food Res Int 33:409-421

El-Saied H, El-Diwany AI, Basta AH, Atwa NA, El-Ghwas DE (2008) Production and characterization of economical bacterial celulose. BioResources 3:1196-1217

Fuller ME, Andaya C, McClay K (2018) Evaluation of ATRFTIR for analysis of bacterial cellulose impurities. J Microbiol Meth 144:145-151

Gallegos AMA, Carrera SH, Parra R, Keshavarz T, Iqbal HM (2016) Bacterial cellulose: a sustainable source to develop value-added products-a review. BioResources 11:5641-5655

Gandini A, Lacerda TM (2015) From monomers to polymers from renewable resources: recent advances. Prog Polym Sci 48:1-39

Gandini A, Lacerda TM (2019) Polymers from plant oils. Scrivener Publishing

Gandini A, Lacerda TM, Carvalho AJF, Trovatti E (2016) Progress of polymers from renewable resources: furans, vegetable oils, and polysaccharides. Chem Rev 116:1637-1669

Gomes FP, Silva NHCS, Trovatti E, Serafim LS, Duarte MF, Silvestre AJD, Pascoal Neto C, Freire CSR (2013) Production of bacterial cellulose by Gluconacetobacter sacchari using dry olive mill residue. Biomass Bioenerg 55:205-211

Higgins HG, Stewart CM, Harrington KJ (1961) Infrared spectra of cellulose and related polysaccharides. J Polym Sci 51:59-84 
Hillmyer MA (2017) The promise of plastics from plants. Science 358:868-870

Hondred PR, Autori C, Kessler MR (2014a) Rare earth triflate initiators in the cationic polymerization of tung oil-based thermosetting polymers for self-healing applications. Macromol Mater Eng 299:1062-1069

Hondred PR, Salat L, Mangler J, Kessler MR (2014b) Tung oilbased thermosetting polymers for self-healing applications. J Appl Polym Sci 131:40406

Huang J, Yuan T, Ye X, Man L, Zhou C, Hu Y, Zhang C, Yang Z (2018) Study on the UV curing behavior of tung oil: mechanism, curing activity and film-forming property. Ind Crops Prod 112:61-69

Huang J, Yuan T, Yang Z, Man L, Hu Y, Yang Z (2019) UV/ thermal dual curing of tung oil-based polymers induced by cationic photoinitiator. Progr Org Coat 126:8-17

Johansson L-S, Tammelin T, Campbell JM, Setälä H, Österberg M (2011) Experimental evidence on medium driven cellulose surface adaptation demonstrated using nanofibrillated cellulose. Soft Matter 7:10917-10924

John G, Nagarajan S, Vemula PK, Silverman JR, Pillai CKS (2019) Natural monomers: a mine for functional and sustainable materials: occurrence, chemical modification and polymerization. Progr Polym Sci 92:158-209

Kawashima N, Yagi T, Kojima K (2019) How do bioplastics and fossil-based plastics play in a circular economy? Macromol Mater Eng 304:1900383

Lacerda TM, Gandini A (2020) The cationic polymerization of tung oil and its fatty-acid methyl ester. Ind Crops Prod 157:112886

Levdik I, Inshakov MD, Misyurova EP, Nikitin VN (1967) Study of pulp structure by infrared spectroscopy. Tr Vses Nauch Issled Irst Tsellyul Bum Prom 52:109-111

Lomège J, Lapinte V, Negrell C, Robin J-J, Caillol S (2019) Fatty acid-based radically polymerizable monomers: from novel poly(meth)acrylates to cutting-edge properties. Biomacromolecules 20:4-26

Madbouly SA, Liu K, Xia Y, Kessler MR (2014) Semi-interpenetrating polymer networks prepared from in situ cationic polymerization of bio-based tung oil with biodegradable polycaprolactone. RSC Adv 4:6710-6718

Manoukian OS, Sardashti N, Stedman T, Gailiunas K, Ojha A, Penalosa A, Mancuso C, Hobert M, Kumbar SG (2019) Biomaterials for tissue engineering and regenerative medicine. Encyclop Biomed Eng 2019:462-482

Meiorin C, Aranguren MI, Mosiewicki MA (2015) Polymeric networks based on tung oil: reaction and modification with green oil monomers. Eur Polym J 67:551-560

Missoum K, Belgacem MN, Bras J (2013) Nanofibrillated cellulose surface modification: a review. Materials 6:1745-1766

Mucci VL, Hormaiztegui MEV, Aranguren MI (2020) Plant oilbased waterborne polyurethanes: a brief review. J Renew Mater 8:579-601

Murawski A, Quirino RL (2019) Bio-based composites with enhanced matrix-reinforcement interactions from the polymerization of $\alpha$-eleostearic acid. Coatings 9:447
O’Donnell A, Dweib MA, Wool RP (2004) Natural fiber composites with plant oil-based resin. Compos Sci Technol 64:1135-1145

Panchal B, Chang T, Qin S, Sun Y, Wang J, Bian K (2020) Optimization and kinetics of tung nut oil transesterification with methanol using novel solid acidic ionic liquid polymer as catalyst for methyl ester synthesis. Renew Energ 151:796-804

Pfister DP, Larock RC (2012) Cationically cured natural oilbased green composites: effect of the natural oil and the agricultural fiber. J Appl Polym Sci 123:1392

Quirino RL, Larock RC (2009) Synthesis and properties of soy hull-reinforced biocomposites from conjugated soybean oil. J Appl Polym Sci 112:2033

Quirino RL, Larock RC (2011) Rice hull biocomposites: Ipreparation of a linseed-oil-based resin reinforced with rice hulls. J Appl Polym Sci 121:2039

Quirino RL, Larock RC (2011b) Rice hull biocomposites, part 2: effect of the resin composition on the properties of the composite. J Appl Polym Sci 121:2050

Quirino RL, Larock RC (2012) Sugarcane bagasse composites from vegetable oils. J Appl Polym Sci 126:860

Quirino RL, Monroe K, Fleischer CH III, Biswas E, Kessler MR (2021) Thermosetting polymers from renewable sources. Polym Int 70:167-180

Ribeiro BO, Valério VS, Gandini A, Lacerda TM (2020) Copolymers of xylan-derived furfuryl alcohol and natural oligomeric tung oil derivatives. Int $\mathrm{J}$ Biol Macromol 164:2497-2511

Sain S, Åkesson D, Skrifvars M (2020a) Synthesis and properties of thermosets from tung oil and furfuryl methacrylate. Polymers 12:258

Sain S, Ảkesson D, Skrifvars M, Roy S (2020b) Hydrophobic shape-memory biocomposites from tung-oil-based bioresin and onion-skin-derived nanocellulose networks. Polymers 12:2470

Sharma V, Kundu PP (2006) Addition polymers from natural oils: a review. Progr Polym Sci 31:983-1008

Sibaja B, Sargent J, Auad ML (2014) Renewable thermoset copolymers from tung oil and natural terpenes. J Appl Polym Sci 131:41155

Tang T, Zhang B, Liu X, Wang W, Chen X, Fei B (2019) Synergistic effects of tung oil and heat treatment on physicochemical properties of bamboo materials. Sci Rep 9:12824

Yoo Y, Youngblood JP (2017) Tung oil wood finishes with improved weathering, durability, and scratch performance by addition of cellulose nanocrystals. ACS Appl Mater Interfaces 9:24936-24946

Zhang C, Garrison TF, Madbouly SA, Kessler MR (2017) Recent advances in vegetable oil-based polymers and their composites. Prog Polym Sci 71:91-143

Publisher's Note Springer Nature remains neutral with regard to jurisdictional claims in published maps and institutional affiliations. 